work of the internbational Opacity Project). The new results obtained are of particular importance for studies of pulsating stars. The atomic data from the Opacity project are available in the database system TOPbase. Some recent work involves the use of those data for the calculation of forces due to radiation pressure.

There have been a number of advances in the calculation of emissivities due to radiative recombination, including new results for may transitions at $U V$, optical and infra-red wavelengths.

\title{
SUMMARY OF THE IRON AND OPACITY PROJECTS
}

\section{KEITH A. BERRINGTON}

\section{Department of Applied Mathematics and Theoretical Physics, Queen's University, Belfast BT7 INN, U.K.}

Considerable effort has been made recently by international collaborations, exploiting advances in atomic physics and in supercomputing, to compute complete sets of accurate data for astrophysically important processes; in particular, the Opacity Project and the IRON Project.

The Opacity Project computed atomic data for opacity calculations

- for H, He, Li, Be, B, C, N, O, F, Ne, Na, Mg, Al, Si, S, Ar, Ca, Fe.

- energies of terms having effective quantum numbers $v \leq 10$ and total angular momentum $L \leq 3$ or 4 , all spin and parity combinations;

- $g$-values for all dipole transitions between these bound terms;

- total cross sections for photoionizaion from all calculated bound terms, tabulated on a grid of photon energies suitable to describe the resonance structure in sufficient detail to calculate reliable opacities;

- line broadening parameters.

28 key research papers arising from the Project, together with calculated energies and oscillator strengths for light ions, are reprinted in 'The Opacity Project Volume 1' (Opacity Project Team, 1994, IOP Publ. ISBN 075030288 7). All data are available from TOPbase, an on-line database at the CDS (Cunto et al. 1993, A\&A 275, L5).

The IRON Project aims to systematically compute electron excitation cross sections for the iron group of elements. Particular attention is given to requirements for the interpretation of data from specific space observations.

In the first stage of the Project excitation cross sections have been computed for fine-structure transitions in the ground configuration of all ions of astrophysical interest. These data are essential for the interpretation of IR lines to be observed by ISO, as well 
as for coronal spectra.

The second stage of the Project, the calculation of excitation rates for all transitions in $\mathrm{Fe}$ and its ions for states with $n \leq 4$, is underway. This will provide data for interpretation of observations from SOHO. Further goals include the calculation of collisional data for nearby elements (e.g. $\mathrm{Ti}, \mathrm{V}, \mathrm{Cr}, \mathrm{Mn}, \mathrm{Co}, \mathrm{Ni}$ ) and of radiative data for fine-structure transitions.

Eventually, the data will be available on-line (TIPbase). Results so far published are in a series entitled 'Atomic data from the IRON Project':

I. Goals and methods. D.G. Hummer, K.A. Berrington, W. Eissner, Anil K. Pradhan, H.E. Saraph, J.A. Tully, 1993, A\&A 279, 298-309

II. Effective collision strengths for infrared transitions in carbon-like ions. D.J. Lennon, V.M. Burke, 1994, A\&AS 103, 273-277

III. Rate coefficients for electron impact excitation of Boron-like ions: $\mathrm{Na}$ VI, $\mathrm{Mg}$ VIII, Al IX, Si X, S XII, Ar XIV, Ca XVI and Fe XXII. Hong Lin Zhang, Mark Graziani, Anil K. Pradhan, 1994, A\&A 283, 319-330

IV. Electron excitation of the ${ }^{2} \mathrm{P}^{\circ}(3 / 2)-{ }^{2} \mathrm{P}^{\circ}(1 / 2)$ fine structure transition in fluorine-like ions. H.E. Saraph and J.A. Tully, 1994, A\&AS (in press)

$V$. Effective collision strengths for transitions in the ground configuration of oxygen-like ions. K. Butler, C.J. Zeippen, 1994, A\&AS (in press)

VI. Collision Strengths and Rate Coefficients for Fe II. Hong Lin Zhang and Anil K. Pradhan, 1994, A\&AS (in press)

VII. Radiative Transition Probabilities for Fe II. S.N. Nahar, 1994, A\&AS

VIII. Electron excitation of the $3 \mathrm{~d}^{4} \mathrm{D}_{\mathrm{J}}$ ground state fine structure transition in the Tilike ions V II, Cr III, Mn IV, Fe V, Co VI and Ni VII. K.A. Berrington, 1994, A\&AS (in press)

IX. Electron excitation of the ${ }^{2} \mathrm{P}^{\circ} 3 / 2-1 / 2$ fine structure transitions in chlorine-like ions from Ar II to Ni XII. J.C. Pelan and K.A. Berrington, 1994, A\&AS (in press)

$X$. Effective collision strengths for infrared transitions in silicon- and sulphur-like ions. M.E. Galavis, C. Mendoza and C.J. Zeippen, 1994, A\&AS

XI. The ${ }^{2} \mathrm{P}_{12-3 / 3}^{\circ}$ fine structure lines of Ar VI, K VII and Ca VIII. H.E. Saraph and P.J. Storey, 1994, A\&AS (submitted) 